\title{
CONVOLUTION INEQUALITIES IN WEIGHTED LORENTZ SPACES
}

\author{
MARTIN KŘEPELA
}

Abstract. We characterize boundedness of a convolution operator with a fixed kernel between the weighted Lorentz spaces $\Lambda^{p}(v)$ and $\Gamma^{q}(w)$ for $0<p \leqslant q \leqslant \infty, 1 \leqslant q<p<\infty$ and $0<$ $q \leqslant p=\infty$. We provide corresponding weighted Young-type inequalities and also study basic properties of some new involved r.i. spaces.

Mathematics subject classification (2010): 44A35, 26D10, 46E30.

Keywords and phrases: Convolution, Young inequality, O'Neil inequality, Lorentz spaces, weights.

\section{REFERENCES}

[1] C. Bennett, R. Sharpley, Interpolation of operators, Pure and Applied Mathematics, 129. Academic Press, Boston, 1988.

[2] A. P. Blozins Ki, Convolution of L $(p, q)$ functions, Proc. Amer. Math. Soc., (32), 237-240, 1972.

[3] M. Carro, A. Gogatishvili, J. Martín, L. Pick, Weighted inequalities involving two Hardy operators with applications to embeddings of function spaces, J. Operator Theory, (59), 309-332, 2008.

[4] M. Carro, L. Pick, J. Soria, V. D. Stepanov, On embeddings between classical Lorentz spaces, Math. Inequal. Appl. (4), 397-428, 2001.

[5] M. CARro, J. Soria, Boundedness of some integral operators, Canad. J. Math., (45), 1155-1166, 1993.

[6] A. Cianchi, L. Pick, Sobolev embeddings into spaces of Campanato, Morrey, and Hölder type, J. Math. Anal. Appl. (282), 128-150, 2003.

[7] M. Ćwikel, A. Kamińska, L. Maligranda, L. Pick, Are generalized Lorentz "spaces" really spaces?, Proc. Amer. Math. Soc., (132), 3615-3625, 2004.

[8] A. Gogatishvili, V. D. Stepanov, Reduction theorems for operators on the cones of monotone functions, J. Math. Anal. Appl., (405), 156-172, 2013.

[9] L. HÖRMANDER, Estimates for translation invariant operators in $L^{p}$ spaces, Acta Math., (104), 93 $140,1960$.

[10] R. A. Hunt, On L( $p, q)$ spaces, Enseign. Math., (12), 249-276, 1966.

[11] A. Kamińska, L. Maligranda, On Lorentz spaces $\Gamma_{p, w}$, Israel J. Math., (140), 285-318, 2004.

[12] A. Kufner, L.-E. Persson, Weighted inequalities of Hardy type, World Scientific Publishing Co., River Edge, 2003.

[13] E. A. Myasnikov, L.-E. Persson, V. D. Stepanov, On the best constants in certain integral inequalities for monotone functions, Acta Sci. Math. (Szeged), (59), 613-624, 1994.

[14] E. Nursultanov, S. Tikhonov, Convolution inequalities in Lorentz spaces, J. Fourier Anal. Appl., (17), 486-505, 2011.

[15] R. Oinarov, Two-sided estimates of the norm of some classes of integral operators, Proc. Steklov Inst. Math., (204), 205-214, 1994.

[16] R. O'NeIL, Convolution operators and L $(p, q)$ spaces, Duke Math. J., (30), 129-142, 1963.

[17] E. SAWYER, Boundedness of classical operators on classical Lorentz spaces, Studia Math., (96), $145-158,1990$.

[18] R. Sharpley, Counterexamples for classical operators on Lorentz-Zygmund spaces, Studia Math., (68), 141-158, 1980.

[19] V. D. Stepanov, The weighted Hardy's inequality for nonincreasing functions, Trans. Amer. Math. Soc., (338), 173-186, 1993. 
[20] L. Y. H. YAP, Some remarks on convolution operators and $L(p, q)$ spaces, Duke Math. J., (36), 647-658, 1969. 